\title{
Cardiac Myocardial Hypertrophy and Altered Swimming Behavior in Xenopus laevis Embryos in Incrementally Increasing Hypergravity
}

\author{
Stacey Howes and Darrell Wiens ${ }^{*}$ \\ Department of Biology, University of Northern Iowa \\ Student: stacey-howes@uiowa.edu \\ *Mentor: darrell.wiens@uni.edu
}

\begin{abstract}
Every living organism on earth has developed and evolved in unit gravity (1G) conditions. It is likely that any deviation from earth's standard gravity will influence development, particularly at early stages. Previous reports from this lab showed that total length is reduced and that ventricle size is increased during development at 7G. The objective of the present study was to investigate the effect of increasing levels of hypergravity on the development of the ventricular myocardial wall and the neuromuscular responsiveness of Xenopus laevis. At early gastrulation, embryos were placed in a centrifuge simulating 7G, 10G, 15G or 17G until they reached stage 45 (feeding tadpole stage, approximately 72 hours from initiation of gastrulation). Mortality was low and only the $17 \mathrm{G}$ exposure induced significant mortality. Immediately following centrifugation, the embryos were stimulated by touching with a probe to test neuromuscular responsiveness. With increasing $\mathrm{G}$ forces, responsiveness to this test was incrementally reduced. A quarter of the embryos were fixed and their body dimensions were measured. One group of remaining live embryos, those exposed to 7G, was maintained and swimming behavior was observed during daily 5 -minute periods for 52 days. Overall, abnormal swimming behavior was found in $33 \%$ of $7 \mathrm{G}$ embryos and in $2 \%$ of controls during this time. These embryos were later subjected to an orientation-swimming test. Hypergravity-exposed embryos required, on average, one second longer ( $9 \%$ of time required) to become oriented. From the embryos exposed to increasing $\mathrm{G}$ levels and then fixed, we found that total length was reduced successively at increasing hypergravity levels, but the snout-vent proportion of total length increased. Stained sections of the fixed embryos revealed that the thickness of the ventricle wall was increased, especially the interior ridge component (trabeculae/papillary muscles). The data demonstrates that hypergravity has persistent effects on the development of the ventricular myocardial wall, neuromuscular responsiveness, and equilibrium organs.
\end{abstract}

\section{KEYWORDS}

Hypergravity, Xenopus, Embryo, Behavior, Myocardium, Hypertrophy 


\section{INTRODUCTION}

Gravity is a universal force on the Earth's surface and every known organism evolved and developed under the same gravitational force and the mechanical stress that it imposes. Because of this constant force, it is likely that many developmental processes rely upon gravity'. Although living things develop under the influence of this force, it is difficult to determine which adaptations and mechanisms are gravity dependent or gravity sensitive. Because of this, research aims manipulate these forces in order to observe the impact on the development of form and physiological processes. There are two manners in which to manipulate these forces: by decreasing force to simulate microgravity (hypogravity) or by increasing force (hypergravity). The research described in this report focuses on hypergravity, but the change of the force in either direction could alter the development differently.

We used the African clawed frog Xenopus laevis as a suitable model organism. Following mating, these amphibians release hundreds of eggs at a time to supply a large number of age-matched subjects of quite similar genetic makeup, thus reducing variability. The embryos are small so that experiments with large numbers are possible. Furthermore, the development time from gastrulation to feeding tadpole is only four days. There is also a large body of knowledge of the progression of embryonic, larval, and adult development. Like all other amphibians, they have a three-chambered heart composed of two atria and one ventricle, distinct from the four-chambered heart of crocodilians, birds and mammals. The muscle in the ventricle of the heart, the myocardium, pumps and circulates the blood and nutrients throughout the body. Gravitational studies have been conducted on several developmental model vertebrates including representative amphibians, birds, and mammals ${ }^{1}$. Though these organisms differ greatly, they share a fundamentally similar development process.

Prior research has shown that early Xenopus embryonic development is altered when subjected to a change in gravity. For example, when early embryos at the beginning of gastrulation were subjected to simulated microgravity via a high aspect ratio bioreactor for five days, they showed changes in morphology compared to the control group including malformations and asymmetries in head cartilages, which are derived from neural crest cells (NCCs) ${ }^{2}$. Following gastrulation, NCCs emerge as the neural tube closes and then migrate, moving to new destinations where they settle and then differentiate into a wide variety of different tissues, including head cartilages. There were also other abnormalities ${ }^{2}$. In earlier studies in which amphibian embryos were subject to microgravity shortly after fertilization (prior to gastrulation) for a short period, there were abnormalities as well, but structure and function returned to normal following return to a $1 \mathrm{G}$ environment $^{3,4}$. Exposure of similarly early Xenopus embryos to hypergravity, on the other hand, has been shown to retard development, induce microcephaly and microphthalmia, suppress gene expression of genes involved in fore- and mid-brain development, and induce abnormal apoptosis in the brain and eyes ${ }^{5}$. In studies involving hypergravity imposed somewhat later in development, conducted by Remus and Wiens ${ }^{6}$ and Duchman and Wiens ${ }^{7}$, embryos were subjected to forces of $7 \mathrm{G}$ or $10 \mathrm{G}$ for five days via centrifugation. Similar to those subjected to microgravity, these larvae showed asymmetries in the head cartilages. There was also evidence of a decrease in total length. However, there did not appear to be any malformations more incapacitating in experimental embryos than in control embryos ${ }^{6,7}$. These authors hypothesized that reduced growth could be the result of a reduced ability to deliver the nutrients of the yolk material because of insufficient cardiovascular circulation. They suggested that this weaker cardiovascular circulation was a result of the increased resistance to circulation imposed by hypergravity and that it would lead to cardiac hypertrophy ${ }^{6,7}$. It is this hypothesis that led to the present study.

Cardiac hypertrophy is the increase in size of the myocardium or cardiac muscle. It is relatively common and arises in response to many cardiac stresses such as arrhythmias, myocardial infarction, hypertension, endocrine disorders, and mechanical load. At first, hypertrophy occurs to compensate for these stresses and to maintain a necessary level of cardiac output. However, sustained 
periods of cardiac hypertrophy can lead to heart fatigue and eventually overall weakness and a decreased stroke volume ${ }^{8}$. The heart's development is early and vital in the developing embryonic circulatory system ${ }^{9}$. Its function provides the mechanism through which nutrients are distributed throughout the body. An overstressed embryonic heart might be predicted to show some signs of hypertrophy as known from the hypertrophied neonatal or adult heart, including increased myofilament size and number, fatigued mitochondria ${ }^{10}$, and deposition of more fibrous extracellular matrix material such as fibronectin ${ }^{11}$. Duchman and Wiens ${ }^{7}$ have recently demonstrated a significant reduction in body cross-sectional area but simultaneously a significant increase in ventricle size in Xenopus embryos subjected to 7G during the early gastrulation to feeding tadpole period ${ }^{7}$. We therefore tested the hypothesis further by studying the morphological features of the myocardial wall in embryos exposed to $7 \mathrm{G}, 10 \mathrm{G}, 15 \mathrm{G}$, and $17 \mathrm{G}$ gravitational fields.

Along with differences in cardiac development, hypergravity is known to induce changes in swimming behavior and orientation ${ }^{12-14}$. In vertebrates, it is the vestibular system that acts to control body, head, and eye posture, as well as swimming behavior. In studies using Xenopus embryos and employing both microgravity and hypergravity, the vestibular system has been examined, and it has been noted that there are significant physiological and behavioral changes ${ }^{12,15}$. These effects lasted long after the termination of the exposure to experimental gravitational forces. Among these effects was an effect on the roll-induced vestibuloocular reflex (rVOR) and another on the spinal motor activity during fictive swimming of the exposed tadpoles ${ }^{12}$. Therefore we investigated the orientation ability and swimming patterns immediately after hypergravity exposure to test the effects of incremental increases in gravity from 1G to 17G. Based on the research of Remus and Wiens $s^{6}$ and Duchman and Wiens ${ }^{7}$, we predicted that hypergravity will induce hypertrophy of the developing myocardial wall with its trabeculae and papillary muscles, increasing their thickness, and that this will be accompanied by an inhibition of body growth as the heart begins to weaken its delivery of nutrients $s^{6,7}$. In addition, we predicted that orientation and swimming behavior would show deviations from normal.

\section{MATERIALS AND METHODS}

\section{a. Embryo Collection}

We produced embryos for experiments by injecting male and female Xenopus laevis frogs with human chorionic gonadotropin, to induce spawning, according to previous protocols ${ }^{16,17}$. Fertilized embryos were sorted with aid of a stereomicroscope based upon viability and arrival at early gastrulation (Nieuwkoop and Faber ${ }^{18}$ stage $10-11$ ). Only viable, normal embryos of the appropriate stage were used for the experiments. Once sorted, the embryos were subjected to $2 \%$ cysteine in $20 \%$ Steinberg's solution (SS) at pH 8.0 for five minutes to completely dejelly them. After dejellying, the embryos were subjected to five washes in $10 \%$ SS for five minutes each. After the final wash, the embryos were sorted again, removing any that were deceased or damaged. Of the remaining viable

embryos, a sample was randomly selected and placed into Costar 24-well trays, each containing $2 \mathrm{~mL}$ of freshly aerated $10 \% \mathrm{SS}$ (pH 7.4) and a substratum pad of $300 \mu \mathrm{L}$ of agarose gel (1\% agarose in 10\% SS). For each experiment there were two trays of control embryos and two trays of experimental embryos (48 in each group, 96 total per experiment). 


\section{b. Exposure to Incrementally Increasing Gravitational Fields}

To simulate hypergravity in the experimental groups, two trays of 24 embryos were placed on the swinging trays on an A-4-62 rotor in an Eppendorf $5810 \mathrm{R}$ centrifuge. The trays were spun for 72 hours at 190 RPM, 220 RPM, 270 RPM, or 300 RPM to yield 7G, 10G, 15G, or 17G, respectively, at the middle of each 24-well tray. The control group trays were taped to the top of the centrifuge chamber to equalize vibration effects. The internal temperature of the centrifuge was monitored and adjusted to provide close consistency between control and experimental groups. Each 24 hours, the centrifuge was stopped for approximately ten minutes to replace the SS with fresh, aerated SS and thus maintain water quality. The 72-hour stopping point was chosen because it is the approximate time required to reach Nieuwkoop and Faber stage 45, the time for normal beginning of feeding. At this point, the neural crest cell derived head skeleton's mandibles and ceratohyals would begin to function and the yolk absorption would quickly diminish. We carried out five experiments, two at 7G, one at 10G, one at 15G, and one at 17G.

Following the completion of the 72-hour experiment, the centrifuge was stopped and all four trays (96 embryos) were removed, the two spun trays from inside the centrifuge and the two $1 \mathrm{G}$ control trays from the top. Any deceased embryos were discarded and mortality rates were recorded. General examination of all larvae in both groups was carried out to observe any differences between control and experimental embryos. Each embryo was lightly touched in the order: tail, trunk, and head. If the embryo responded (noted by starting to swim or a change in swimming direction or fictive swimming), the result was recorded and the embryo was not poked in any other location. Each embryo was touched until a response was recorded or 'no response' was recorded. From each set of embryos, five were removed and flash frozen for future study and ten were fixed in $4 \%$ paraformaldehyde for various studies. The remaining embryos of each group were kept in separate 250 $\mathrm{mL}$ dishes for studies of behavior and observation of any long-term hypergravity effects.

\section{c. Behavior Studies}

Live larvae were observed for five minutes daily for 52 days to record any abnormal swimming that might occur. During abnormal swimming, embryos often oriented upside down while moving short distances, swam in tight circles, or swam without making progress. Each larva was scored as simply demonstrating aberrant swimming behavior or not, and the percentage within the $1 \mathrm{G}$ and $7 \mathrm{G}$ exposed groups was recorded daily. We also carried out orientation tests at one month after centrifugation to observe any delays or difficulties. This test was performed once comparing only the $7 \mathrm{G}$ exposed group to its $1 \mathrm{G}$ control group. The larvae were placed one at a time into a $50 \mathrm{~mL}$ graduate cylinder containing about $40 \mathrm{ml}$ SS. The cylinder was covered with Parafilm and then quickly inverted twice and returned to upright, and the tadpole was timed to determine its ability to orient and swim to the bottom. Normally all tadpoles first seek the bottom following disorientation. Each day during the swimming behavior observations, any deceased embryos were discarded and were recorded to determine if there was a difference in mortality rates.

\section{d. Fixation, Embedment and Sectioning}

After fixation in $4 \%$ paraformaldehyde the larvae were measured to determine total length and snout-to-vent length using a binocular dissecting microscope. After fixation and measurements, they were dehydrated in an ascending ethanol series, cleared in Protocol Safeclear II (Fisher Scientific) and embedded in paraffin. They were next individually mounted on wooden blocks, trimmed and sectioned at six $\mu \mathrm{m}$ onto $42^{\circ} \mathrm{C}$ water on Tissue Section Adhesive (Richard Allen Scientific, Kalamazoo, MI) coated slides using a slide warmer to allow the sections to dry onto the slides. The slides were deparaffinized in xylene, rehydrated, and then stained with hematoxylin and eosin and mounted under coverslips in GVA mount (Invitrogen). In some cases the sections were mounted without staining for differential interference contrast viewing.

\section{AJUR volume 12 | Issue 2 | January 2015}


Total Body

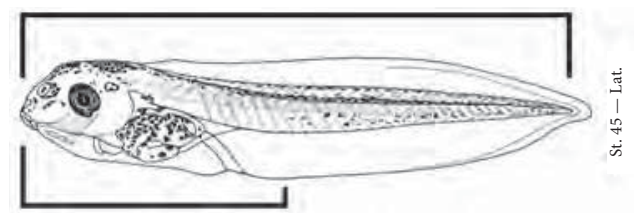

Snout-vent

\section{e. Dimensional Analysis of Sections}

The mounted transverse sections were viewed and photographed with a Leica DMIRE 2 microscope using bright field and differential interference contrast optics. Using image analysis software, (Image J, National Institutes of Health) the sections were analyzed and the thickness of the ventricle wall was measured. In addition, papillary muscle thickness and the area of their extension into the ventricle chamber were also measured. Multiple samples from each embryo were collected to find the averages. The image software was recalibrated before each use to insure accuracy.

\section{f. Statistical Analysis of Data}

Once all measurements for the embryos were made, SAS (Statistical Analysis System) Software was used to conduct Shapiro-Wilk normality tests on all data sets. SAS was then used to perform two-sample t-tests on the corresponding control and experimental data sets. From each experiment, measurements were collected from hypergravity and control groups and recorded. Following data collection, a statistical standardization was completed in order to normalize differences between the various experimental groups. This was achieved by first comparing and analyzing all control groups and then finding an averaged measurement. Next, the average difference in size of a control group was calculated and recorded as a percentage. Finally, the difference in average size was applied to the corresponding experimental group for a normalized set of data across all experimental groups. Outliers remained in collected data but were not used in calculations. Significance probabilities of all measurements were completed and recorded. Significance was indicated in the data if the probability was 0.05 or less. In addition, the standard deviation was calculated for all groups and displayed in the appropriate figure. 


\section{RESULTS}

We used a tabletop centrifuge to expose Xenopus embryos at early gastrulation stage to gravitational fields of $7 \mathrm{G}, 10 \mathrm{G}, 15 \mathrm{G}$, and $17 \mathrm{G}$, along with $1 \mathrm{G}$ controls. The exposure lasted three days until the feeding tadpole stage. During this period, most of the organs come into being, beginning with the heart. In all of these experiments we observed that mortality was quite low: $4-6 \%$ in the control, 7G, 10G, and 15G groups. There were no significant differences among them. However, at $17 \mathrm{G}$ the mortality was $100 \%$. Thus we found a clear limit to the amount of $\mathrm{G}$ force the embryos could survive.

\section{a. Embryo Responsiveness, Swimming Behavior, and Orientation Ability}

The neuromuscular responsiveness of the larvae immediately following the termination of centrifugation was investigated by gently stimulating with a probe. By lightly prodding with a probe the tail, trunk, and head region in sequence we determined the first touch that elicited a response. The data are shown in Table 1. We found that Xenopus tadpoles are quite sensitive and were very likely to respond. Most $1 \mathrm{G}$ (control) tadpoles responded to the minimum stimulation, a tail touch, and only $1 \%$ did not respond to any stimulus. Experimental embryos showed decreasing responsiveness, which required stimulation more and more anteriorly, and this loss of responsiveness was gravity-dependant. The proportion showing no response, for example, increased incrementally from $1 \%$ to almost $12 \%$ as $\mathrm{G}$ force increased from $1 \mathrm{G}$ through 7,10 , and $15 \mathrm{G}$ (Table 1). The differences between control and each experimental group were statistically significant.

\begin{tabular}{|c|c|c|c|c|}
\hline \multicolumn{5}{|c|}{ Table 1. Embryo Touch Test Responsiveness* } \\
\hline & Control & $7 \mathrm{G}$ & $10 \mathrm{G}$ & 15G \\
\hline Tail touch & $91.4 \%$ & $75.0 \%$ & $63.6 \%$ & $42.9 \%$ \\
\hline Trunk & $5.7 \%$ & $16.7 \%$ & $22.7 \%$ & $35.7 \%$ \\
\hline Head & $1.9 \%$ & $4.2 \%$ & $6.8 \%$ & $9.5 \%$ \\
\hline No Response & $1.0 \%$ & $4.1 \%$ & $6.9 \%$ & $11.9 \%$ \\
\hline Total & $100.0 \%$ & $100.0 \%$ & $100.0 \%$ & $100.0 \%$ \\
\hline
\end{tabular}

We also made long-term observations of swimming behavior. We accumulated this data over a period of almost eight weeks by simply recording whether or not aberrant swimming was seen in individuals that had been exposed to 7G occupying a $250 \mathrm{ml}$ glass dish, during a period of five minutes. The percentage of individuals in the group showing aberrant swimming; such as swimming upside-down, swimming in tight circles, or swimming without progress; was calculated. These data are shown in Figure 1. On all but one day (day 18 post-experiment) the tadpoles exposed to $7 \mathrm{G}$ showed $15-80 \%$ incidence of aberrant swimming. Such swimming was observed among control tadpoles on only seven of the 52 days and was seen in never more than $10 \%$ of the individuals in the group. 


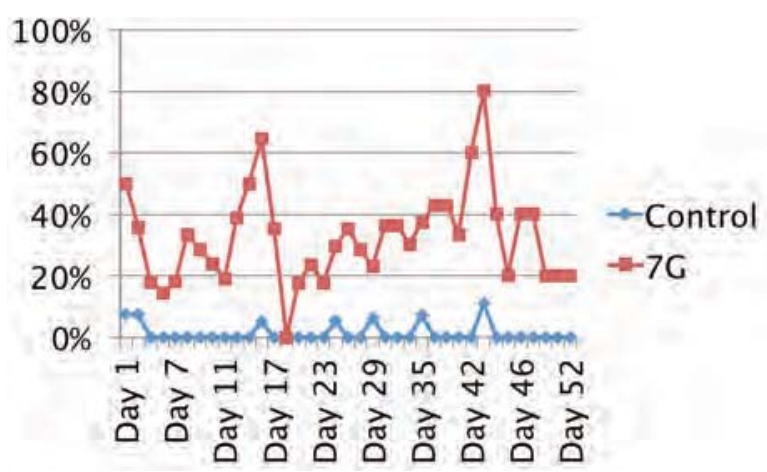

Figure 1. Aberrant swimming behavior among control and 7G exposed Xenopus tadpoles. During five minutes of observation on successive days, the percent of individuals showing odd swimming was calculated. Those that exhibited such behavior were recorded and removed from the well to prevent double counting. The percentage of $7 \mathrm{G}$ tadpoles showing aberrant swimming was significantly greater on all days but one. The behavior was far more frequent in the hypergravity-exposed group.

As a third method to evaluate neuromuscular behavior development in hypergravity, we tested the ability of the 7G-exposed tadpoles to orient after a disrupting turbulence in a column of water. This testing was carried out one month after the hypergravity exposure. Xenopus larvae normally seek the bottom in a vessel of water after disturbance of orientation. Testing one individual at a time, we induced the disturbance and quickly timed the arrival to the bottom surface, as described in Materials and Methods. The results are displayed in Figure 2. The distribution of individuals timed showed that the quickest orienting tadpoles required only a second among controls, but almost two seconds among those exposed to $7 \mathrm{G}$ hypergravity. There was a consistent delay of almost a second through the sample trials of more than 40 tadpoles.

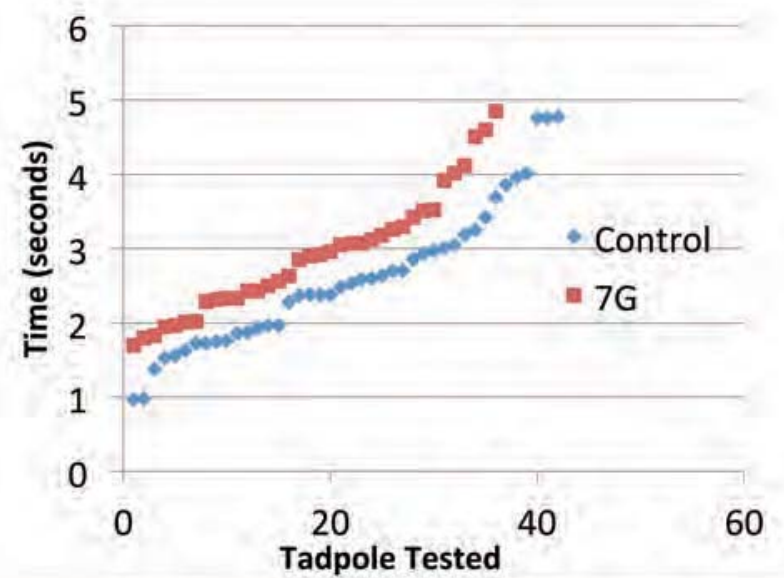

Figure 2. Scatter distribution of times required to orient in a disturbed water column and seek and arrive at the bottom. Number along the $\mathrm{X}$-axis shows individuals tested. The quickest orienting individuals required about one second among controls, but almost two seconds among the $7 \mathrm{G}$ exposed. There was a delay of almost a second through the sample of more than 40 tadpoles.

\section{b. Body Length Measurements}

To verify the inhibition of growth in body length in hypergravity demonstrated in previous work in this $l a b^{6,7}$, and to test the gravity-dependence of this, we obtained total body and snout-to-vent lengths, and calculated the ratios of these for all four magnitudes of G-force. The comparisons were 
made from normalized data (as described in Materials and Methods) because the size of embryos varied from experiment to experiment due to parent genetic factors (and perhaps other) factors. We found that statistically significant differences in overall total length did exist between each experimental group and the control group. Although the difference between $10 \mathrm{G}$ and $15 \mathrm{G}$ groups was not significant, the difference between the $10 \mathrm{G}$ and $7 \mathrm{G}$ groups was. Tadpole total length was found, on average, to be reduced as G-force increased (Table 2).

\begin{tabular}{|c|c|c|c|c|}
\hline \multicolumn{5}{|c|}{ Table 2. Body Lengths of Tadpoles Exposed to Increasing Gravitational Force } \\
\hline & Control (1G) & 7 G & 10G & 15G \\
\hline $\begin{array}{c}\text { Mean Body } \\
\text { Length } \pm \text { SD (mm) }\end{array}$ & $8.18 \pm 0.21$ & $7.69^{*} \pm 0.54$ & $6.88^{*} \pm 0.51$ & $6.56^{*} \pm 0.99$ \\
\hline $\begin{array}{c}\text { Mean Snout-vent } \\
\text { Length } \pm \text { SD (mm) }\end{array}$ & $3.26 \pm 0.10$ & $3.27 \pm 0.22$ & $3.24 \pm 0.23$ & $3.29^{* *} \pm 0.27$ \\
\hline \multicolumn{7}{|c|}{ Sample Size } & 80 & 20 & 20 & 20 \\
\hline${ }^{*}$ Significantly shorter than control. ${ }^{*}$ Significantly longer than control. \\
\hline
\end{tabular}

In contrast, average snout-to-vent length was not reduced by hypergravity. The differences among the four groups were small and not significant, with exception of the 15G group, which were actually slightly but significantly longer controls (Table 2). Because this result indicates that the effect of hypergravity on body length was mainly to reduce the tail, we plotted the proportion of total length occupied by the head-trunk as a function of G-force. It is evident from the graph (Figure 3) that this ratio responded to increasing gravity with corresponding incremental increases.

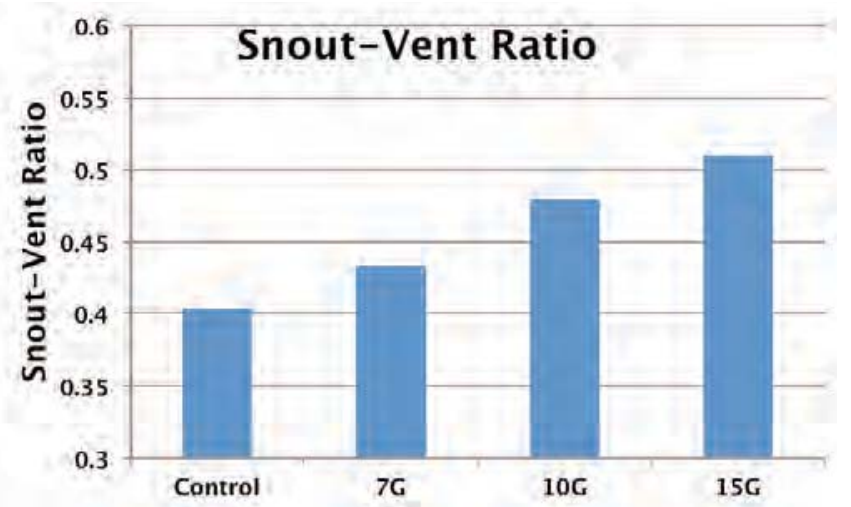

Figure 3. The proportion of the body composed of snout-vent (the head with trunk length as a percentage of total length) increased in parallel with an increase in gravitational force during development. Thus the region containing the enlarged heart and nearby vital organs grew more than the peripheral propulsion organ, the tail.

\section{c. Cross Sectional Width of Ventricle Wall}

In order to explore the cardiac hypertrophy reported earlier by Duchman and Wiens ${ }^{7}$, we sectioned some fixed and embedded tadpoles from each group transversely through the heart and histologically stained the sections or viewed them using differential interference microscopy. From calibrated images of these we measured ventricle wall thickness, and the length and area of the muscle ridges (trabeculae or papillary muscles) extending into the chamber from the wall. The measurements were made as indicated in Figure 4. 


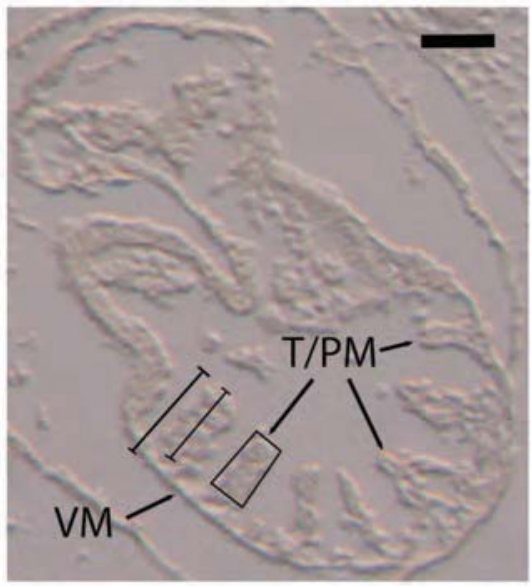

Figure 4. Transverse section through control tadpole revealing the heart and measurements taken. The image was captured using differential interference microscopy. The ventricular myocardium (VM) wall is of normal thickness and the trabeculae / papillary muscles (TPM) extend into the ventricle chamber prominently. We measured the thickness of the ventricle wall from outside edge to continuous cavity space (longer bracketed line) in several locations, the lengths of the several trabeculae / papillary muscles (shorter bracketed line), and also their areas (rectangle). Bar $=50 \mu \mathrm{m}$.

We observed no statistically significant differences in ventricle wall thickness in the $7 \mathrm{G}$ group compared to controls. However, we did find significant differences between the controls and the $10 \mathrm{G}$ and $15 \mathrm{G}$ groups. The ventricle wall was thicker, approximately a third thicker than controls in the $15 \mathrm{G}$ group (Figure $5 \mathrm{C}$ ). The thickness appeared to increase as $\mathrm{G}$-force increased. The data for these measurements are shown in Figure 5.
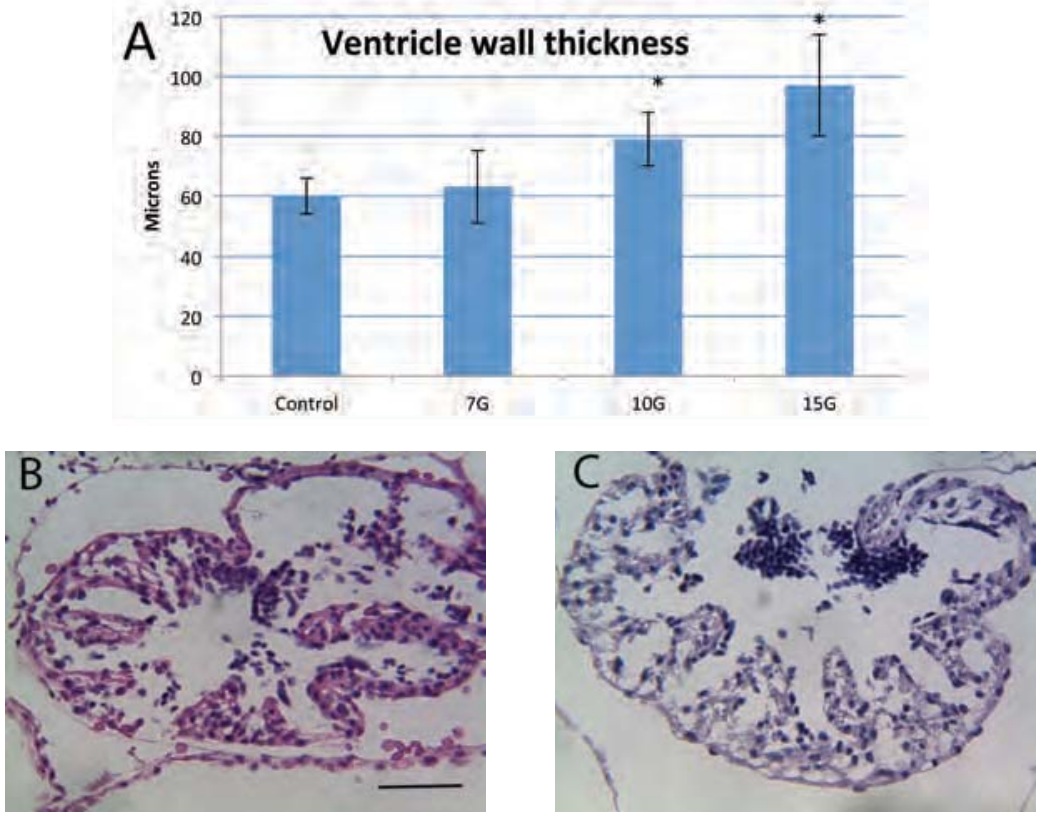

Figure 5. Ventricle wall thickness and hypergravity. A. Thickness was measured from the outer surface of myocardium to the continuous cavity region inside (includes trabeculae / papillary muscles), among all populations of control and experimental larvae. The numbers of walls measured were 135 for control, and 45 each for the $7 \mathrm{G}, 10 \mathrm{G}$, and $15 \mathrm{G}$ groups. Error bars indicate standard deviations. B. Section through heart of control embryo. C. Section through heart of embryo exposed to 15G. Size and prominent muscular ridges show evidence of hypertrophy. Bar $=50 \mu \mathrm{m}$.

*Significantly thicker than control group. 


\section{d. Cross Sectional Areas of Cardiac Trabeculae/Papillary Muscles}

Because the muscle ridges that occupy the interior of the myocardial walls develop early as the forthcoming trabeculae, and the papillary muscles attaching to valves, it is possible they also become more prominent during hypertrophy. We were unable to distinguish trabeculae from papillary muscles in sections, thus we considered them together. We evaluated their size by using the imaging software to draw approximate rectangles over their profiles extending into the cavity in the sections and we calculated the areas. The results are shown in Figure 6. We found statistically significant differences in these mean areas compared to control groups as a result of developing in hypergravity. The mean areas were $16 \%, 30 \%$, and $90 \%$ greater in $7 \mathrm{G}, 10 \mathrm{G}$ and $15 \mathrm{G}$ experimental groups. As with ventricle wall thickness, these increased incrementally with increasing G-force.

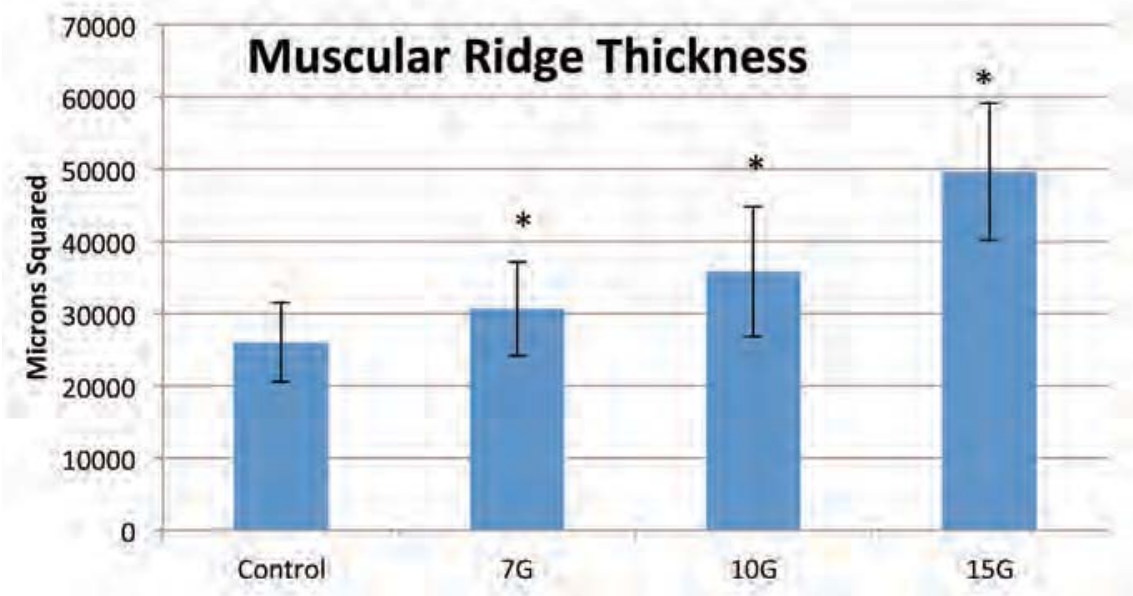

Figure 6. The areas in sections of the muscular ridges extending interiorly from the ventricular myocardial wall inward (trabeculae and papillary muscles) were measured among all populations of control and hypergravity-exposed tadpoles. The bars represent the mean of these areas with standard deviations shown as error bars. The numbers of ridges measured were 135 for control, and 45 each for the $7 \mathrm{G}, 10 \mathrm{G}$, and $15 \mathrm{G}$ groups. The mean areas in sections were incrementally increased in $7 \mathrm{G}, 10 \mathrm{G}$ and $15 \mathrm{G}$ experimental groups. ${ }^{*}$ Significantly greater than control.

\section{DISCUSSION}

In this study we employed centrifugation to simulate hypergravity and test its effects on Xenopus laevis embryonic development from early gastrulation (Nieuwkoop and Faber stage 10 - 11) to stage 45, when feeding begins. This period encompasses the time of development of the heart (its formation is apparent at stage $29-30$ and contractions begin at stage 33-34) and also the other major organs. We simulated gravitational fields of $7 \mathrm{G}, 10 \mathrm{G}, 15 \mathrm{G}$, and $17 \mathrm{G}$, along with $1 \mathrm{G}$ controls during each centrifugation, and we made observations and measurements of body and heart dimensions to see if they would confirm and extend previous findings $s^{6,7}$ and elucidate whether the effects increase with incremental increases in gravitational force. In the experiments at $7 \mathrm{G}$, we also evaluated the development of swimming and orientation ability. Thus we investigated in greater detail the growth inhibition and cardiac hypertrophy effects seen previously ${ }^{6,7}$ and we also looked for evidence of effects on the organs of balance, swimming and orientation.

In all of our experiments the embryos began their development and continued as far as gastrulation in unit gravity $(1 \mathrm{G})$. Thus they bypassed the effects that hypergravity is known to have on earlier development. This includes effects on the development of signaling centers and inductions, on cleavage and its spindle positioning, and on involution, which begins formation of germ layers. Therefore our findings relate only to the effects of hypergravity on organogenesis rather than on early events. 
We noted that the mortality rate was low compared to that observed in previous work from this $\mathrm{lab}^{7}$. We attribute this to more stringent screening and selection of embryos for the experiments, and perhaps to the good health status of the parent frogs. Based on this low mortality it is apparent that the forces associated with even $15 \mathrm{G}$ can be tolerated remarkably well by Xenopus once they have made it as far as gastrulation at unit gravity. They emerged from the centrifuge looking smaller but basically normal, and most were able to respond to stimulation and swim briefly. However, the centrifugation at $17 \mathrm{G}$ did result in complete mortality. This indicates the maximum gravitational stress load that these embryos can withstand lies between 15G and 17G (for our experimental set-up).

Our results from the touch testing for responsiveness (testing was done shortly after the end of centrifugation) showed that responsiveness, very high at unit gravity, declined with increasing G-force. Larvae required touches at increasingly anterior locations to show a response, and the proportion responding declined. Responding to a stimulus with swimming minimally involves functional receptors, neural integration, and muscle contraction. Our observations are a predictable outcome if gravitational force has an impact on components of this system. Several studies have provided evidence for such effects. Böser and Horn ${ }^{12}$ showed that ventral root motor activity recorded during fictive swimming (tail motion producing swimming bursts) in Xenopus is sensitive to development in $3 \mathrm{G}$ hypergravity, and that recorded mean burst duration increased significantly. Furthermore, Horn and Sebastian ${ }^{19}$ and Pronych et. al. ${ }^{20}$ reported that the vestibular system reflex rVOR is sensitive to hypergravity during a sensitive period, indicating that oriented swimming motion can be affected. The rVOR depends on the maculae and cristae to help the animal control posture and response to visual stimuli. In addition, Horn et. al. ${ }^{21}$ reported evidence of a microgravity exposure effect on the shape of otoconia and on the number and size of Calbindin-expressing cells in the maculae. Thus hypergravity might be predicted to also have effects. Our results showing an incremental increase in inhibition of responsiveness from control to $7 \mathrm{G}, 10 \mathrm{G}$, and $15 \mathrm{G}$ and also showing that $7 \mathrm{G}$ hypergravity-exposed tadpoles required significantly more time to gain orientation and swim to the bottom after induced turbulence, add new information for Xenopus. In a 1998 study Fejtek et. al. ${ }^{14}$ reported no substantive effects of hypergravity exposure on swimming behavior in Xenopus tadpoles. However in their experiments only one exposure, at 3G, was used. Because the embryos in our study handled up to $15 \mathrm{G}$ and were still able to feed and swim, $3 \mathrm{G}$ is probably not sufficient to alter swimming behavior, but our results show that 7G and higher forces do alter both the incidence of aberrant swimming and swimming orientation ability.

In previous reports from our lab, total length and snout-to-vent length were found to be stunted by development over the same period (gastrulation through feeding tadpole stage) in $7 \mathrm{G}$ hypergravity ${ }^{7}$ and both $7 \mathrm{G}$ and $10 \mathrm{G}$ hypergravity ${ }^{6}$. Furthermore, the effect at $10 \mathrm{G}$ was greater than the effect at $7 \mathrm{G}^{6}$. In the present study we found that the average total length declined commensurately with increasing gravitational force, as expected. This supports the hypothesis of an incremental effect of G-force. However, the snout-to-vent length measurements were not found to be different. The embryos had quite similar snout-to-vent length through all gravitational levels. But in the present study the data were normalized (as described in Materials and Methods) in order to correct for variability in the size of embryos from experiment to experiment due to genetic factors. It is probable that snout-to-vent lengths would have also been similar in the previous studies if the normalization had been applied. In the current study we found that the proportion of the total length that was composed of head and trunk in fact became greater as the level of gravity increased (Figure 3), even though total length decreased. This implies a decreased amount of total length composed of the tail. This is consistent with the hypothesis that a hypertrophied and weakening heart is less able to distribute nutrients throughout the entirety of the body, particularly in higher gravitational forces. Duchman and Wiens did measure the body cross-section area of the trunk and found that $7 \mathrm{G}$ hypergravity caused it to decrease $\mathrm{P}^{7}$. Therefore it seems that hypergravity causes a narrowing of the trunk even though its length becomes an increasing proportion of total length. 
This may be related to insufficient nutrient distribution.

We also measured histological properties of the heart. Though the ventricle wall was not significantly thicker, the interior ridges (trabeculae and papillary muscle lengths and areas) increased significantly with gravitational force. These findings extend those of Duchman and Wiens ${ }^{7}$ who found that the cross-sectional area of the heart was increased by exposure to 7G. If the ventricular myocardium is thicker and has longer and broader ridges, this difference at least partly accounts for the larger cross-sectional area. Taken together with body size and proportion data, this can be taken as further support of cardiac hypertrophy and insufficient circulatory support for tail growth.

The initiation and early development of the beating heart is the most vital component in the circulation of blood. Furthermore, the heart is responsible for the dispersion of nutrients throughout the body from the yolk mass via the developing circulatory system. The increase of gravitational force adds a resistance to the dispersion of the blood with its nutrients and therefore places an increased load on the heart. Our data support the idea that this increased workload and cardiac stress result in cardiac hypertrophy, particularly in the ventricle, as a response to gravitational stress. The hindered ability of the heart to pump nutrients throughout the rest of the body may have an inhibitory effect on growth, especially in tail length, and could affect swimming ability because the tail is the primary locomotor organ. This effect could augment the likely effects on development of balance organs and reflexes such as rVOR.

We have provided new information and insight into the effect of hypergravity upon the development of the ventricular myocardial wall, neuromuscular responsiveness and equilibrium organs. Further study should seek biochemical or physiological evidence through the use of marker peptides such as atrial natriuretic factor (ANF) or fibronectin (FN). ANF is a cardiac hypertrophy modulator secreted at higher levels during hypertrophy. FN has been found to participate in regulating the orientation of synthesized collagen and therefore myocardial compliance. Myocardial compliance is an important feature that is usually decreased in the presence of cardiac hypertrophy. Furthermore, FN levels may also be higher in areas of cardiac damage, a symptom of cardiac hypertrophy, as it accumulates in these areas. FN levels and orientation could be investigated using an antibody to show its immunohistological features in hypergravity-exposed hearts.

\section{ACKNOWLEDGEMENTS}

We wish to acknowledge the University of Northern Iowa College of Humanities, Arts and Sciences for funding and support of this research. We thank Jessica Moon, Director of the University Honors Program, for advice and support, Dr. Ira Simet, Professor of Chemistry and Biochemistry for critical reading and advice, and Carl Reitz for help with sectioning and staining of tissues.

\section{REFERENCES}

[1] Morey-Holton, E. (2003) Evolution on Planet Earth: Impact of the Physical Environment, 1st ed., Elsevier, Amsterdam.

[2] Olson, W. M., Wiens, D. J., Gaul, T. L., Rodriguez, M., and Hauptmeier, C. L. (2010) Xenopus development from late gastrulation to feeding tadpole in simulated microgravity, The international journal of developmental biology 54, 167-174.

[3] Duprat, A. M., Husson, D., and Gualandris-Parisot, L. (1998) Does gravity influence the early stages of the development of the nervous system in an amphibian?, Brain research. Brain research reviews $28,19-24$. 
[4] Souza, K. A., Black, S. D., and Wassersug, R. J. (1995) Amphibian development in the virtual absence of gravity, Proc Natl Acad Sci USA 92, 1975-1978.

[5] Kawakami, S., Kashiwagi, K., Furuno, N., Yamashita, M., and Kashiwagi, A. (2006) Effects of hypergravity environments on amphibian development, gene expression and apoptosis, Comparative biochemistry and physiology. Part A, Molecular \& integrative physiology 145, 65-72.

[6] Remus, R., and Wiens, D. J. (2008) The Effects of hypergravity on the morphology of Xenopus embryos, American Journal of Undergraduate Research 7, 19-26.

[7] Duchman, B., and Wiens, D. J. (2011) Effects of hypergravity on Xenopus embryo growth and cardiac hypertrophy, American Journal of Undergraduate Research 11, 1-12.

[8] Frey, N., and Olson, E. N. (2003) Cardiac hypertrophy: the good, the bad, and the ugly, Annual review of physiology $65,45-79$.

[9] Gilbert, S. (2014) Developmental Biology, 10th ed., Sinauer Associates Inc, Sunderland, MA.

[10] Goldstein, M. A., Cheng, J., and Schroeter, J. P. (1998) The effects of increased gravity and microgravity on cardiac morphology, Aviation, space, and environmental medicine 69, A12-16.

[11] Mamuya, W. S., and Brecher, P. (1992) Fibronectin expression in the normal and hypertrophic rat heart, The Journal of clinical investigation 89, 392-401.

[12] Böser, S., and Horn, E. R. (2006) Hypergravity susceptibility of ventral root activity during fictive swimming in tadpoles (Xenopus laevis), Archives italiennes de biologie 144, 99-113.

[13] Horn, E., and Sebastian, C. (1996) A hypergravity related sensitive period during the development of the roll induced vestibuloocular reflex in an amphibian (Xenopus laevis), Neuroscience letters 216, $25-28$.

[14] Fejtek, M., Souza, K. A., Neff, A., and Wassersug, R. J. (1998) Swimming kinematics and respiratory behavior of Xenopus laevis larvae raised in altered gravity, Journal of Experimental Biology 201, $1917-1926$.

[15] Kashiwagi, A., Hanada, H., Kawakami, S., Kubo, H., Shinkai, T., Fujii, H., and Kashiwagi, K. (2003) Effects of high gravity on amphibian development, Uchu Seibutsu Kagaku 17, 215-216.

[16] Etheridge, A., and Richter, S. (1978) Xenopus laevis: Rearing and breeding the African Clawed Frog, NASCO Publishing Agencies, Ft. Atkinson, WI.

[17] Wu, M., and Gerhart, J. (1991) Raising Xenopus in the laboratory, Methods in cell biology 36, 3-18.

[18] Nieuwkoop, P., and Faber, J. (1967) Normal table of development of Xenopus laevis. (Daudin), North-Holland Publications, Amsterdam.

[19] Sebastian, C. E., Pfau, K., and Horn, E. R. (1998) An age-dependent sensitivity of the roll-induced vestibuloocular reflex to hypergravity exposure of several days in an amphibian (Xenopus laevis), Acta astronautica 42, $419-430$.

[20] Pronych, S., Souza, K. A., Neff, A., and Wassersug, R. J. (1996) Optomotor behavior in Xenopus laevis tadpoles as a measure of the effect of gravity on visual and vestibular neural integration, Journal of Experimental Biology 199, 2689 - 2701.

[21] Horn, E., Böser, S., Membre, H., Dournon, C., Husson, D., and Gualandris-Parisot, L. (2006) Morphometric investigations of sensory vestibular structures in tadpoles (Xenopus laevis) after a spaceflight: implications for microgravity-induced alterations of the vestibuloocular reflex, Protoplasma 229, 193-203. 


\section{ABOUT THE STUDENT AUTHOR}

Stacey Howes is from Fairfax, lowa and graduated from the University of Northern lowa (UNI) in May, 2014. At UNI she majored in biology and carried out this research for her University and Biology Honors thesis. She submitted an abstract describing aspects of this work for presentation at the 2013 annual meeting of the American Society for Space and Gravitational Research in New Orleans, Louisiana. Her abstract was accepted and at the meeting she presented a poster and was also invited to give a talk. She received recognition for one of the best talks given by an undergraduate student at the meeting. Stacey is now pursuing studies at the University of lowa College of Dentistry.

\section{PRESS SUMMARY}

Life on earth has developed and evolved in unit gravity (1G) conditions and it is likely that any deviation in this force would affect early development. The objective of this study was to investigate the effect of increasing levels of gravitational force on the development of body dimensions, the cardiac muscular wall and the neuromuscular responsiveness of the African Clawed frog Xenopus laevis. Early embryos were placed in a centrifuge simulating 7G, 10G, 15G or 17G until they reached the feeding tadpole stage, and then they were examined and tested. We found that with increasing $G$ forces, responsiveness was incrementally reduced, aberrant swimming behavior was more frequent, and orientation ability was slower. Their total length was reduced successively at increasing hypergravity levels, but the snout-vent proportion of total length increased. The thickness of the ventricle wall was increased, especially the interior ridges, suggesting hypertrophy. Our data demonstrate that hypergravity has persistent effects on the development of the ventricular myocardial wall, neuromuscular responsiveness, and equilibrium organs.

\section{AJUR volume 12 | Issue 2 | January 2015}

